УДК 616.3-008.1

DOI: 10.18101/2306-1995-2019-2-44-51

\title{
ЧАСТОТА ВСТРЕЧАЕМОСТИ ПЕРЕКРЕСТОВ ФУНКЦИОНАЛЬНЫХ ЗАБОЛЕВАНИЙ ЖЕЛУДОЧНО-КИШЕЧНОГО ТРАКТА
}

\section{(C) Найданова Эржена Гармаевна}

кандидат медицинских наук, доцент, Бурятский государственный университет им. Д. Банзарова Россия, 670000, г. Улан-Удэ, ул. Октябрьская, 36

E-mail: erg60@mail.ru

Функциональные заболевания желудочно-кишечного тракта (ФЗ ЖКТ) наиболее актуальная сфера современной гастроэнтерологии и являются широко распространенными заболеваниями в мире. ФЗ ЖКТ в общемировой популяции страдают 15-25\% взрослого населения развитых стран. В настоящее время недостаточно изучены все патогенетические звенья.

В РК (Римском консенсусе) IV Комитет экспертов охарактеризовал ФЗ ЖКТ как группу расстройств, протекающих с нарушением взаимодействия ЦНС (головной мозг) и периферического звена нервной системы (ось «головной мозг-ЖКТ»). Патогенез ФЗ ЖКТ недостаточно исследован. Выявлено, что симптомы функциональных заболеваний нередко сочетаются между собой (перекрещиваются). Эпидемиологические исследования указывают разные типы «перекрестов» Ф3 ЖКТ. Перекрестные симптомы более разнообразно сочетаются у пациентов более молодого возраста. Изучение перекрестных симптомов функциональных заболеваний позволит создать более комплексный подход к лечению этих заболеваний.

Ключевые слова: желудочно-кишечный тракт, функциональные заболевания, синдром раздраженного кишечника, функциональная диспепсия, перекрестные симптомы, молодой возраст.

Для цитирования:

Найданова Э. Г. Частота встречаемости перекрестов функциональных заболеваний желудочно-кишечного тракта // Вестник Бурятского государственного университета. Медицина и фармация. 2019. Вып. 2. С. 44-51.

Функциональные заболевания желудочно-кишечного тракта (ФЗ ЖКТ) - наиболее актуальная сфера современной гастроэнтерологии и являются широко распространенными заболеваниями в мире. ФЗ ЖКТ в общемировой популяции страдают $15-25 \%$ взрослого населения развитых стран $[1,10,11,13]$. В настоящее время недостаточно изучены все патогенетические звенья ФЗ [1-4].

Хотя ФЗ ЖКТ не представляют непосредственной угрозы для жизни больных они чаще развиваются у людей работоспособного возраста и значительно снижают качество жизни $[1,2,5,6]$. Разнообразная симптоматика, частые рецидивы способствуют проведению диагностических мероприятий, что приводит к дополнительным экономическим потерям $[6,8,9]$. Таким образом, Ф3 ЖКТ можно отнести к социально значимым заболеваниям. 
Э. Г. Найданова. Частота встречаемости перекрестов функциональных заболеваний желудочно-кишечного тракта

В 2016 г. Римским консенсусом IV предложена классификация Ф3 ЖКТ:

- расстройства функции пищевода,

- расстройства функции кишечника,

-расстройства центрального генеза, проявляющиеся абдоминальной болью,

- расстройства функции желчного пузыря и сфинктера Одди,

- аноректальные расстройства.

В РК IV Комитет экспертов охарактеризовал ФЗ ЖКТ как группу расстройств, протекающих с нарушением взаимодействия ЦНС (головной мозг) и периферического звена нервной системы (ось «головной мозг-ЖКТ») [10].

Патогенез ФЗ ЖКТ недостаточно исследован. Выявлено, что симптомы функциональных заболеваний нередко сочетаются между собой (перекрещиваются). Эпидемиологические исследования указывают разные типы «перекрестов» ФЗ ЖКТ. Например, частота признаков синдрома раздраженного кишечника (СРК) среди больных с функциональной диспепсией (ФД) составляет 46\%, а у $33,8 \%$ больных с ФД наблюдаются симптомы рефлюкса [2,3]. В этих случаях встает вопрос о ведущей патологии для проведения необходимого обследования и лечения [8, 9]. В 2011 году Ghoshal и соавт. выявили, что частота СРК среди больных с ФД варьирует от 1,6\% до 49, 0\%. Также, в 2005 году при исследовании 700 взрослых выявлены ФД у 44,8\%, ГЭРБ (гастроэзофагеальная рефлюксная болезнь) у 34,45\%, СРК у 19,4\% исследуемых. При чем, перекрест всех 3-х заболеваний отмечен у $13,3 \%$ пациентов [12].

Цель исследования: определить частоту перекрестов функциональных заболеваний желудочно-кишечного тракта у лиц различного возраста.

\section{Материалы и методы}

В исследовании использовались анкеты, составленные ФГБУ НИИ МПС СО РАМН (зав. отделением В. В. Цуканов, д-р мед. наук, проф.). Вопросы анкеты составлены с учетом диагностики СРК, ФД, функциональной изжоги, функционального запора. Предварительные диагнозы функциональных заболеваний выставлялись исходя из PK IV $[7,9]$.

\section{Результаты и их обсуждение}

Симптомы СРК чаще проявляются в молодом возрасте: у $11 \%$ у студентов и $8 \%$ у «взрослых» (табл. 1) [7]. 


\section{Частота проявлений симптомов ФЗ ЖКТ}

\begin{tabular}{|c|c|c|}
\hline $\begin{array}{c}\text { Вид функционального } \\
\text { заболевания ЖКТ }\end{array}$ & $\begin{array}{c}\text { Частота } \\
\text { проявлений } \\
\text { студентов (в \%) }\end{array}$ & $\begin{array}{c}\text { Частота проявления } \\
\text { у «взрослых» (в \%) }\end{array}$ \\
\hline ФД & 5,5 & 6 \\
\hline СРК & 11 & 8 \\
\hline Запоры & 11 & 12 \\
\hline Изжога & 15,1 & 18 \\
\hline
\end{tabular}

Также, выявлено, что у студентов с СРК чаще, чем у «взрослых» встречаются симптомы: вздутие живота, диспепсия, изжога, срыгивание, боли в правом и левом подреберье (студенты к «взрослым» соответственно 100:75,0; 62,5:50,0;50,0:25,0;62,5:50,0). У «взрослых» чаще выявляется связь болей с стрессом и ощущения кислоты и горечи в горле (75,0:50,0; 75,0:50,0 соответственно) (табл. 2) [7].

Таблий 2

Сопутствующие симптомы у больных с проявлениями СРК (в \%)

\begin{tabular}{|c|c|c|c|c|c|c|c|c|c|}
\hline 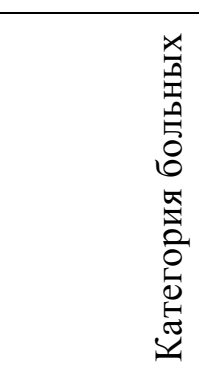 & $\begin{array}{l}\overline{0} \\
\tilde{\tilde{J}} \\
\overline{\tilde{O}} \\
\dot{0}\end{array}$ & 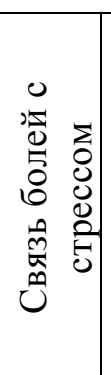 & 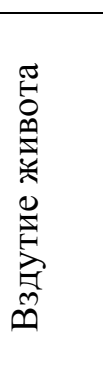 & 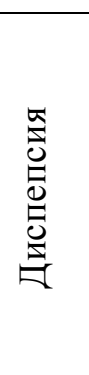 & 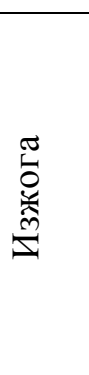 & 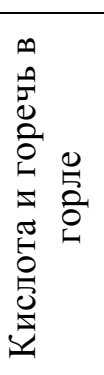 & 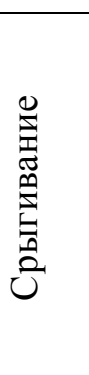 & 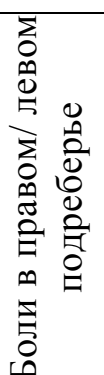 & 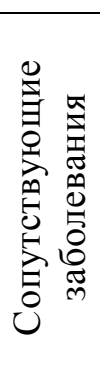 \\
\hline Студенты & 20,8 & 50,0 & 100,0 & 62,5 & 62,5 & 50,0 & 50,0 & 62,5 & 50,0 \\
\hline Взрослые & 48,3 & 75.0 & 75,0 & 50.0 & 50,0 & 75,0 & 25,0 & 50.0 & 50.0 \\
\hline Итого & 29,9 & 58,3 & 91,2 & 58,3 & 58,3 & 58,3 & 41,7 & 58,3 & 50,0 \\
\hline
\end{tabular}

У больных с ФД боли в низу живота, изжога, срыгивание чаще встречаются у лиц более молодого возраста (соотношение студенты и взрослые соответственно 75,3:33,3; 100,0:33,3; 75,0:33,3). Лица более старшего возраста чаще предъявляют жалобы на ощущения кислоты и горечи в горле, боли в правом или левом подреберье (взрослые и студенты соответственно 66,7:50,0; 66,7:50,0) (табл. 3) [7]. 
Э. Г. Найданова. Частота встречаемости перекрестов функциональных заболеваний желудочно-кишечного тракта

Таблицุа 3

Сопутствующие заболевания и симптомы у больных с ФД (в \%)

\begin{tabular}{|c|c|c|c|c|c|c|c|c|c|}
\hline 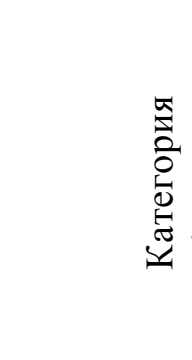 & 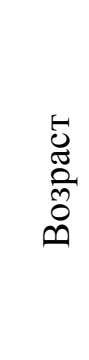 & 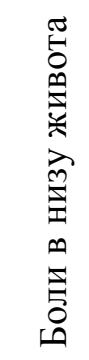 & 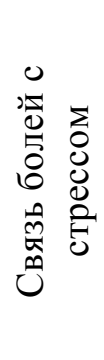 & 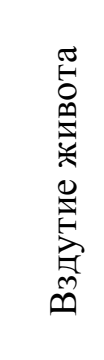 & 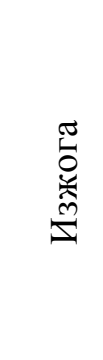 & 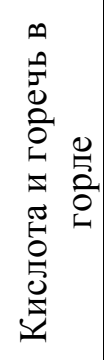 & 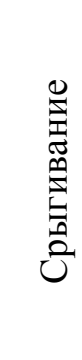 & 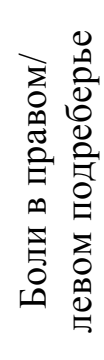 & 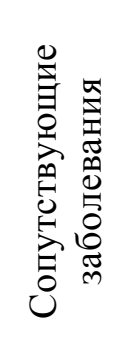 \\
\hline Студенты & 20,8 & 75,0 & $\begin{array}{c}100, \\
0\end{array}$ & 100,0 & 100,0 & 50,0 & 75,0 & 50,0 & 100,0 \\
\hline Взрослые & 55,3 &, 3 & - & & 33,3 & 66,7 & 33,3 & 66,7 & 100,0 \\
\hline Итого & 35,6 & 57,1 & 57,1 & 57,1 & 71,4 & 57,1 & 57,1 & 57,1 & 100,0 \\
\hline
\end{tabular}

У молодых людей с изжогой такие симптомы как боли в низу живота, диспепсия, ощущение кислоты и горечи в горле, срыгивание встречаются чаще, чем у людей старшего возраста $(63,6: 22,2 ; 72,7: 66,7 ; 81,8: 66,7 ; 72,7: 33,3$ соответственно) (табл. 4). В то время, как у «взрослых» чаще встречаются боли в правом или левом подреберье (77,8:36,4 соответственно) [7].

Таблицьа 4

Сопутствующие заболевания и симптомы у больных с изжогой (в \%)

\begin{tabular}{|c|c|c|c|c|c|c|c|c|c|}
\hline 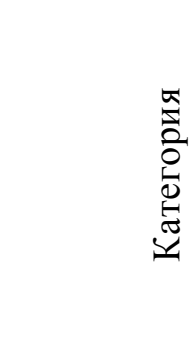 & 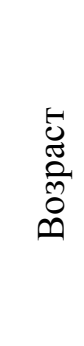 & 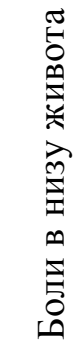 & 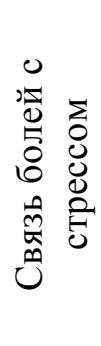 & 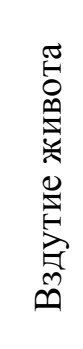 & 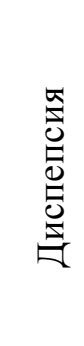 & 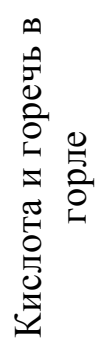 & 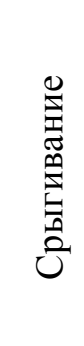 & 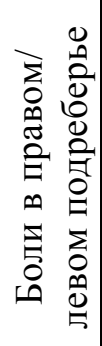 & 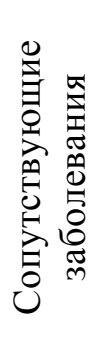 \\
\hline Студенты & 19,8 & 63,6 & 27,3 & 72,7 & 72,7 & 81,8 & 72,7 & 36,4 & 72,3 \\
\hline Взрослые & 44,2 & 22,2 & 22,2 & 77,8 & 66,7 & 66,7 & 33,3 & 77,8 & 77,8 \\
\hline Итого & 32,7 & 45,0 & 25,0 & 75,0 & 70,0 & 75,0 & 55,0 & 55,0 & 75,0 \\
\hline
\end{tabular}

Если рассмотреть больных с запорами, то такие симптомы, как боли в низу живота, связь болей с стрессом, вздутие живота, изжога, срыгивание чаще встречаются у лиц более молодого возраста $(62,5: 33,3 ; 50,0: 33,3$; $62,5: 33,3 ; 50,0: 16,7)$ (табл. 4). У «взрослых» пациентов чаще наблюдаются боли в правом или левом подреберье (77,8:36,4 соответственно) [7]. 
Таблица 5

Сопутствующие заболевания и симптомы у больных с запорами (в \%)

\begin{tabular}{|c|c|c|c|c|c|c|c|c|c|c|}
\hline 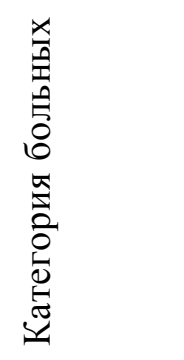 & $\begin{array}{l}5 \\
\tilde{\tilde{J}} \\
\tilde{\tilde{O}} \\
\stackrel{0}{0}\end{array}$ & 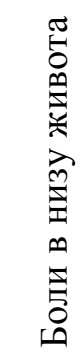 & 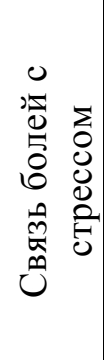 & 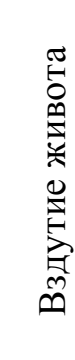 & 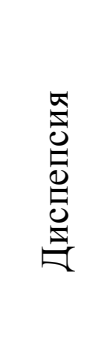 & 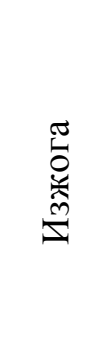 & 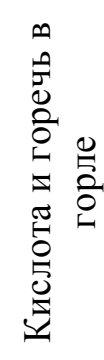 & 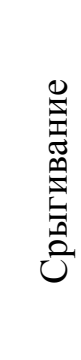 & 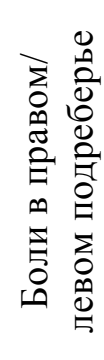 & 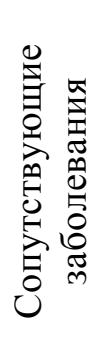 \\
\hline Студ & 9,9 & 62,5 & 50,0 & 75,0 & 37,5 & 62,5 & 50,0 & 50,0 & 37,5 & 62,5 \\
\hline Взрослые & 43,7 & 33,3 & 33,3 & 83,3 & 50,0 & 33,3 & 50,0 & 16,7 & 50,0 & 50,0 \\
\hline Итого & 30,8 & 50.0 & 42,9 & 78,6 & 42,9 & 50,0 & 50,0 & 35.7 & 42,9 & $\overline{57,1}$ \\
\hline
\end{tabular}

Также, при исследовании выявлено, что форма ФД у студентов чаще всего представлена в виде СЭБ (синдрома эпигастральной боли), в то время, как у взрослых встречается как СЭБ так и ППДС (постпрандиальный дистресс синдром) (66,7\% и 33,3\% соответственно). Частота сочетания СРК с ФД составляет $37,5 \%$ у студентов и $25,0 \%$ у «взрослых», ФД с СРК составляет $75 \%$ у студентов и $33,3 \%$ - у «взрослых» (табл. 6) [7].

\section{Частота сочетания СРК с ФД и ФД с СРК}

\begin{tabular}{|c|c|c|}
\hline $\begin{array}{c}\text { Категория } \\
\text { больных }\end{array}$ & $\begin{array}{c}\text { Синдром раздражённого } \\
\text { кишечника с ФД (в \%) }\end{array}$ & $\begin{array}{c}\text { Функциональная диспепсия } \\
\text { с СРК (в \%) }\end{array}$ \\
\hline Студенты & 37,5 & 75,0 \\
\hline Взрослые & 25,0 & 33,3 \\
\hline Всего & 33,3 & 57,1 \\
\hline
\end{tabular}

\section{Выводы}

Симптомы СРК чаще проявляются в молодом возрасте (у 11\% студентов и $8 \%$ у «взрослых»).

У молодых людей с СРК чаще, чем у более старших пациентов встречаются симптомы: вздутие живота, диспепсия, изжога, срыгивание, боли в правом и левом подреберье (студенты к «взрослым» соответственно 100:75,0; 62,5:50,0; 50,0:25,0; 62,5:50,0).

У молодых людей с ФД боли в низу живота, изжога, срыгивание чаще встречаются, чем у лиц более старшего возраста (соотношение студенты и «взрослые» соответственно 75,3:33,3; 100,0:33,3; 75,0:33,3).

У молодых людей с изжогой такие симптомы как боли в низу живота, диспепсия, ощущение кислоты и горечи в горле, срыгивание 
Э. Г. Найданова. Частота встречаемости перекрестов функциональных заболеваний желудочно-кишечного тракта

встречаются чаще, чем у людей старшего возраста $(63,6: 22,2 ; 72,7: 66,7$; $81,8: 66,7 ; 72,7: 33,3$ соответственно).

У молодых людей с запорами такие симптомы, как боли в низу живота, связь болей с стрессом, вздутие живота, изжога, срыгивание чаще встречаются у лиц более молодого возраста $(62,5: 33,3 ; 50,0: 33,3$; $62,5: 33,3 ; 50,0: 16,7)$.

У «взрослых» пациентов с изжогой чаще наблюдаются боли в правом или левом подреберье (77,8:36,4 соответственно).

Лица более старшего возраста с ФД чаще предъявляют жалобы на ощущения кислоты и горечи в горле, боли в правом или левом подреберье («взрослые» и студенты соответственно 66,7:50,0; 66,7:50,0).

Форма ФД у студентов чаще всего представлена в виде СЭБ (синдрома эпигастральной боли), в то время, как у «взрослых» встречается как СЭБ так и ППДС (постпрандиальный дистресс синдром) (66,7\% и 33,3\% соответственно). Частота сочетания СРК с ФД составляет $37,5 \%$ у студентов и $25,0 \%$ у «взрослых», ФД с СРК составляет $75 \%$ у студентов и $33,3 \%$ - у «взрослых».

Перекрестные симптомы более разнообразно сочетаются у пациентов более молодого возраста. Изучение перекрестных симптомов функциональных заболеваний позволит создать более комплексный подход к лечению этих заболеваний.

\section{Лuтература}

1. Эволюция представлений о функциональных заболеваниях желудочнокишечного тракта в свете Римских критериев IV пересмотра (2016 г.) / Д. Н. Андреев [и др.] // Российский журнал гастроэнтерологии гепатологии колопроктологии. 2017. № 27(1). С. 4-11.

2. Бельмер С. В., Хавкин А. И., Печкуров Д. В. Функциональные расстройства органов пищеварения у детей (в свете Римских критериев IV). М.: Ремдер. 2016. 40 с.

3. Клинические рекомендации Российской гастроэнтерологической ассоциации и Ассоциации колопроктологов России по диагностике и лечению синдрома раздраженного кишечника / В. Т. Ивашкин [и др.] // Российский журнал гастроэнтерологии гепатологии и колопроктологии. 2017. № 27(5). C. 76-93.

4. Клинические рекомендации Российской гастроэнтерологической ассоциации по диагностике и лечению функциональной диспепсии / В. Т. Ивашкин [и др.] // Российский журнал гастроэнтерологии гепатологии и колопроктологии. 2017. № 27(1). С. 450-61.

5. Клинические проявления у детей школьного возраста, имеющие множественные комбинированные гастроинтестинальные симптомы / Д. Ю. Латышев [и др.] // ЭНИ Забайкальский медицинский вестник. 2017. № 3. С. 36-45.

6. Функциональная диспепсия в свете Римских критериев IV пересмотра (2016 г.) [Электронный ресурс] / И. В. Маев [и др.] // Гастроэнтерология. 2016. № $\quad 2 . \quad$ http://con-med.ru/magazines/gastroenterology/gastroenterology-02- 
2016/funktsionalnaya_dispepsiya_v_svete_rimskikh_kriteriev_iv_peresmotra _2016_g (дата обращения: 20.02.2019).

7. Найданова Э. Г., Спасова Т. Е., Григорьева Е. В. Сравнительный анализ перекрестных симптомов функциональных заболеваний желудочнокишечного тракта // Современная наука: актуальные проблемы теории и практики. 2018. № 10. С. 87-92.

8. Пиманов С. И., Силивончик Н. Н. Римские IV рекомендации по диагностике и лечению функциональных гастроэнтерологических расстройств. M., 2016. $160 \mathrm{c}$.

9. Canavan C., West J., Card T. Review article: The economic impact of the irritable bowel syndrome // Aliment Pharmacol Ther. 2014. № 40(9). C. 1023-1034.

10. Devanarayana N. M., Radjindrajith S., Benninga M. A. Quality of life and health care consultation in 13 to 18 year olds with abdominal pain predominant functional gastrointestinal diseases // BMC Gastroenterol. 2014. № 14. C. 150.

11. Lawrence S. Friedman, Laurence J. Brandt. Sleisenger and Fordtran's Gastrointestinaland Liver Disease: Pathophysiology, Diagnosis, Management // Edited by Mark Feldman 10th ed. 2015. 2616 c.

12. Papatheodoridis G. V., Karamanolis D. G. Prevalence and impact of upper and lower gastrointestinal symptoms in the Greek urban general population // Scand J Gastroenterol. 2005. № 40. C. 412-421.

13. Pediatric Quality of Life Inventory (PedsQL) Gastrointestinal Symptoms Module Testing Study Consortium. Healthrelated quality of life in pediatric patients with functional and organic gastrointestinal diseases / J.W. Varni [et al.] // J Pediatr. 2015. № 166(1). C. 85-90. 
Э. Г. Найданова. Частота встречаемости перекрестов функциональных заболеваний желудочно-кишечного тракта

\section{INCIDENCE OF FUNCTIONAL DISEASE CROSSOVER GASTROINTESTINAL}

\section{Erthzena G. Naydanova}

Candidate of Medical Sciences, Associate Professor

of the Department of Therapy

Medical Institute, Dorzhi Banzarov Buryat State University

36a, Oktyabrskaya st., Ulan-Ude, 670001, Russia

E-mail: erg60@mail.ru

Functional diseases of the gastrointestinal tract (FZ GIT) — the most relevant area of modern gastroenterology and are widespread diseases in the world. FG gastrointestinal tract in the global population affects $15-25 \%$ of the adult population in developed countries. Currently, all pathogenetic links are not well understood.

In RK IV (Rome consensus) the Expert Committee characterized the gastrointestinal tract as a group of disorders that occur with impaired interaction of the central nervous system (brain) and the peripheral link of the nervous system (axis "brain — gastrointestinal tract"). The pathogenesis of gastrointestinal tract F. is not well understood. It was revealed that the symptoms of functional diseases are often combined (intersect). Epidemiological studies indicate different types of "crossroads" of gastrointestinal tract. Cross-symptoms are more diverse in younger patients. The study of the cross-symptoms of functional diseases will create a more comprehensive approach to the treatment of these diseases.

Keywords: gastrointestinal tract, functional diseases, irritable bowel syndrome, functional dyspepsia, cross-over symptoms, young age. 